\title{
Adsorbed or intercalated: Na on graphene/Ir(111)
}

\author{
Petar Pervan ${ }^{1, *}$ and Predrag Lazić $2, \dagger$ \\ ${ }^{1}$ Institute of Physics, Bijenička 46, HR-10000 Zagreb, Croatia \\ ${ }^{2}$ Rudjer Boskovic Institute, Bijenička 54, HR-10000 Zagreb, Croatia
}

(Received 21 June 2017; published 7 September 2017)

\begin{abstract}
Interaction of sodium with graphene $(\mathrm{Gr})$ on $\operatorname{Ir}(111)$ was studied with the aim to resolve the issue of $\mathrm{Na}$ adsorption/intercalation kinetics. The system $\mathrm{Na} / \mathrm{Gr} / \mathrm{Ir}(111)$ was studied by means of angle-resolved photoemission spectroscopy, low-energy electron diffraction, and ab initio density functional theory (DFT) calculation. It has been found that at room temperature (RT) and low concentrations Na is dominantly adsorbed on graphene. At higher concentrations, an intercalation process sets in so that it is possible to observe the coexistence of these two states. Eventually, all $\mathrm{Na}$ atoms are found in the intercalated state as determined by exposure to oxygen. While adsorption of $\mathrm{Na}$ on graphene already intercalated by $\mathrm{Na}[\mathrm{Na} / \mathrm{Gr} / \mathrm{Na} / \mathrm{Ir}(111)$ system] at RT was not possible, we could observe Li adsorption through the increase of Dirac point binding energy. Li coadsorption strongly affects the binding energy of the iridium surface state as well. This finding was supported by DFT calculations of adsorption energy of $\mathrm{Na}$ and Li on bare and fully $\mathrm{Na}$ intercalated graphene.
\end{abstract}

DOI: 10.1103/PhysRevMaterials.1.044202

\section{INTRODUCTION}

Despite the extraordinary physical properties of graphene, there are several reasons why, from the early days of graphene research, scientists have been trying to alter its structural and electronic properties [1] as well as its interaction with the supporting surfaces [2-4]. Chemical functionalization of graphene was widely used in order to tune its electronic properties [5,6] and to introduce a band gap at the Fermi level $[1,3,7]$. In the same vein, it has been demonstrated that adsorption and/or intercalation of alkali metals (AMs) could be successfully used to modify the charge-carrier density in graphene, manipulate its electronic structure [8,9], and thereby alter its chemical reactivity [10-12].

In some cases, AM adsorption/intercalation results in electron transfer to graphene without any significant alteration of its electronic structure $[13,14]$. The outcome of such AM application is reportedly a virtually rigid shift of the graphene $\pi$ bands [15]. By contrast, some other systems show a clear departure from the rigid band shift [16]. Also, the intercalation of graphene by AMs is known to alter its collective electronic properties [9,14,17,18]. A doping-induced shift of the Dirac point to higher binding energies is usually associated with the band renormalization just below the Fermi level which, in turn, is due to the electron-phonon coupling (EPC) [16,19]. The EPC enhancement is expected to induce the superconductive state $[17,20,21]$, as it has been realized for many other carbon-based materials [22]. The unique electronic structure of graphene appears to be very sensitive to its coupling to the substrate to the point of total extinction of characteristic Dirac-like dispersion of $\pi$ bands around the $K$ point. Coupling to the

\footnotetext{
*pervan@ifs.hr

†plazic@irb.hr
}

Published by the American Physical Society under the terms of the Creative Commons Attribution 4.0 International license. Further distribution of this work must maintain attribution to the author(s) and the published article's title, journal citation, and DOI. surface is particularly strong for graphene on Ni [23], Co [24], and $\mathrm{Ru}$ [25]. Intercalation of AM can lead to a large increase in the graphene/substrate separation with the effect of graphene decoupling from the substrate accompanied by the recovery of the $\pi$ band's dispersion [2,26-28].

It is known from the previous extensive research on graphite [29-32] that intercalation of graphene as one of several carbon allotropes is by no means a straightforward process. Graphene with a densely structured two-dimensional mesh appears to be impenetrable for almost all atoms, including hydrogen [33]. Whether particular atoms deposited on graphene will intercalate or not depends on many factors such as intercalation vs adsorption bonding energy, temperature, graphene microstructure (i.e., the existence of wrinkles), etc. $[34,35]$.

While the density functional theory (DFT) calculations almost unanimously indicate that intercalation is an energetically preferable state for all AM atoms with respect to adsorption on graphene [36], the experimental evidence shows a more complex picture [16]. A recent DFT study of AM adsorption/intercalation on graphene supported by the $\mathrm{Au} / \mathrm{Ni}(111)$ substrate showed a substantially larger binding energy of all AM in the intercalated state than in the adsorbed state [36]. Li apparently has the strongest affinity for intercalation against adsorption with an energy difference of around $1 \mathrm{eV}$ [36]. On the other hand, x-ray photoemission spectroscopy and angle-resolved photoemission spectroscopy (ARPES) study of the AM position with respect to graphene (adsorption vs intercalation) on a one-monolayer (ML) $\mathrm{Au} / \mathrm{Ni}(111)$ substrate implies that only $\mathrm{K}$ and $\mathrm{Ca}$ intercalate into the $\mathrm{Gr} / \mathrm{Au}$ interface, whereas $\mathrm{Cs}, \mathrm{Rb}, \mathrm{Na}$, and $\mathrm{Li}$ adsorb preferably on top of the graphene layer [16]. This result extends the earlier finding of easy intercalation of graphene on $\mathrm{Au} / \mathrm{Ni}(111)$ by $\mathrm{K}$ reported by Varykhalov et al. [3] as well.

In contrast to the $1-\mathrm{ML} \mathrm{Au} / \mathrm{Ni}(111)$ substrate, it seems that graphene on other substrate surfaces (metallic and $\mathrm{SiC}$ ) is readily intercalated by $\mathrm{Li}$ [37-39]. Potassium showed a similar behavior regarding the intercalation of graphene on $\operatorname{Ir}(111)$ $[19,40,41]$ and $\mathrm{Ni}(111)[4,42]$ at the room temperature (RT). 
It appears that $\mathrm{Cs}$ intercalates graphene on $\mathrm{Ni}(111)$ [42] and Ir (111) [35] surfaces at RT as well. However, a combined scanning tunnel microscopy (STM), low electron energy microscopy, and ARPES study of Cs intercalation of graphene on $\operatorname{Ir}(111)$ has shown that the process follows a somewhat more complicated path [35]. At submonolayer coverages, Cs displays the coexistence of adsorbed and intercalated phases (see Fig. 2 in Ref. [35]) such that the intercalation process is in a delicate energetic balance relative to adsorption, which is due to van der Waals interaction between graphene and the iridium surface. Although this has not been explicitly discussed, it appears as if $\mathrm{K}$ interaction with $\mathrm{Gr} / \mathrm{Ir}(111)$ exhibits a similar coexistence of adsorbed and intercalated phases [see Fig. 2(a) in Ref. [40]] at the initial K coverage.

Sodium appears to be an exception when it comes to AM intercalation of graphite; that is, it is the only AM that does not intercalate [32,43]. Different aspects of Na intercalation of graphene on metal surfaces are still being debated. Park et al. [26] reported a complete recovery of the electronic structure of graphene from the strongly interacting Ni surface by spontaneous $\mathrm{Na}$ intercalation at RT. Even more recent experiments by Matyba et al. [2] have convincingly demonstrated that $\mathrm{Na}$ adsorbs preferably on graphene on $\mathrm{Ni}(111)$ at $\mathrm{RT}$ and that intercalation takes place only at elevated temperatures. This is explained in terms of the increased mobility of $\mathrm{Na}$ atoms, suggesting that the diffusion of adsorbed $\mathrm{Na}$ from the external graphene surface to the intercalated configuration is controlled by kinetic effects, e.g., penetration through graphene defects (i.e., the wrinkles at grain boundaries) [35].

STM and ARPES investigations of Na interaction with graphene on $\operatorname{Ir}(111)$ imply that $\mathrm{Na}$ does not intercalate but rather remains adsorbed; initially, it decorates the step edges, whereas at higher coverages it forms single-layer islands [7]. Such an adsorbed layer of $\mathrm{Na}$ was reported to downshift the Dirac point to $1.5 \mathrm{eV}$ below the Fermi level. On the other hand, Gall et al. [44] have found that $\mathrm{Na}$, and likewise $\mathrm{K}$ and $\mathrm{Cs}$, intercalates graphene on $\operatorname{Ir}(111)$ in the temperature interval 300-700 K, as verified by Auger electron spectroscopy and thermal desorption spectroscopy. Finally, Langer et al. [45] concluded in their study of sheet plasmons in bare and $\mathrm{Na}$ intercalated graphene on $\operatorname{Ir}(111)$ that $\mathrm{Na}$ intercalates fully at RT.

The aim of this work is to contribute to the resolution of the dilemma over $\mathrm{Na}$ adsorption vs intercalation of graphene (Gr) on $\operatorname{Ir}(111)$ through a detailed ARPES and DFT study of this system. We show that, initially, Na preferentially adsorbs on $\mathrm{Gr} / \mathrm{Ir}(111)$ at RT, but with further exposure, we observe signatures of $\mathrm{Na}$ intercalation. Finally, after long exposures all the $\mathrm{Na}$ atoms become intercalated, and no indications of adsorbed Na were found. Moreover, we are able to demonstrate that the large shift of the Dirac point to higher binding energies is associated with intercalation and not with an adsorbed state of $\mathrm{Na}$ atoms. We also show that once the graphene layer is intercalated by $\mathrm{Na}$, no extra $\mathrm{Na}$ adsorption on top of graphene is possible, while $\mathrm{Li}$ adsorption (under the same conditions) proves possible, leading to an additional charge transfer to graphene and to a further shift of the Dirac point to higher binding energies. The experiments were corroborated by the DFT calculations of adsorption energies of $\mathrm{Na}$ and $\mathrm{Li}$ on bare and intercalated graphene.

\section{EXPERIMENTAL AND COMPUTATIONAL DETAILS}

Experiments were performed in an ultrahigh-vacuum ARPES facility. The ARPES spectra were recorded by a Scienta SES100 hemispherical electron analyzer with an overall energy resolution of $25 \mathrm{meV}$ and an angular resolution of $0.2^{\circ}$. Photons of $21.2 \mathrm{eV}$ from a nonpolarized He-discharge ultraviolet source (beam-spot diameter of around $2 \mathrm{~mm}$ ) were used for excitation.

An iridium single crystal of $99.99 \%$ purity and orientation accuracy better than $0.1^{\circ}$ was used. The substrate was cleaned by several cycles of sputtering with $1.5 \mathrm{keV} \mathrm{Ar}^{+}$ions at room or elevated temperature $(1100 \mathrm{~K})$ followed by annealing at 1500 $1600 \mathrm{~K}$. The cleanliness and quality of $\operatorname{Ir}(111)$ were checked by low-energy electron diffraction and ARPES (surface-state sharpness and intensity [46]). The graphene monolayer on $\operatorname{Ir}(111)$ was prepared by a temperature-programmed growth cycle (TPG; room-temperature ethene exposure $6 \times 10^{-6} \mathrm{~Pa}$ for $60 \mathrm{~s}$ and flash to $1400 \mathrm{~K}$ ) followed by chemical vapor deposition (CVD; $6 \times 10^{-6} \mathrm{~Pa}$ of ethene for $300 \mathrm{~s}$ while the sample was held at $1150 \mathrm{~K}$ ). This TPG + CVD procedure growth leads to uniform orientation of graphene with the lattice aligned to the substrate lattice (R0) and at full monolayer coverage [47]. Sodium was deposited from a commercial getter source while the sample surface was kept at room temperature. The extremely reactive sodium adatoms, particularly at higher coverages when a metallic layer is formed, are susceptible to contamination from the residual gas molecules $[10,48]$. The experiments were prepared and performed by taking all the necessary steps to ensure that the experimental results were not affected by undesired adsorption; that is, they were carried out at low base pressure during $\mathrm{Na}$ deposition and data acquisition.

Ab initio calculations were performed within the framework of DFT $[49,50]$ as implemented in the VASP code $[51,52]$ with the projector augmented-wave method [53,54]. We used a selfconsistently implemented van der Waals density functional (vdW-DF) [55,56] for correlation in combination with the optimized Becke88 (optB88) for the exchange energy [57]. The lattice constant of the Ir bulk was determined selfconsistently, while the graphene lattice constant was adjusted to match the $\operatorname{Ir}(111)$ surface lattice constant, resulting in some strain $(\sim 10 \%)$ on the graphene. This makes graphene somewhat more reactive in binding to $\mathrm{Li}$ and $\mathrm{Na}$. However, the relative strength of the binding is still correct.

In all calculations, the expansion in plane waves was done using the cutoff energy of $500 \mathrm{eV}$. The Brillouin zone was sampled by a Monkhorst-Pack [58] choice of $3 \times 3 \times 1 k$ points. The $\operatorname{Ir}(111)$ surface slab was simulated by five atomic layers, of which the top two, along with the $\mathrm{C}$ and $\mathrm{Li} / \mathrm{Na}$ atoms, were allowed to relax until the forces on atoms were below $1 \mathrm{meV} / \AA$. In all calculations, the size of the surface unit cell was $4 \times 4$ (for Ir unit cells, the graphene unit cell was expanded by about $10 \%$ to fit commensurably). Such a structure accommodates a maximum of 16 intercalated and 16 adsorbed $\mathrm{Li} / \mathrm{Na}$ atoms. Different coverages were simulated by randomly distributing a selected number of $\mathrm{Li} / \mathrm{Na}$ atoms. Dipole correction $[59,60]$ in the direction perpendicular to the slab was used with $20 \AA$ of vacuum separating the periodic slab images. 

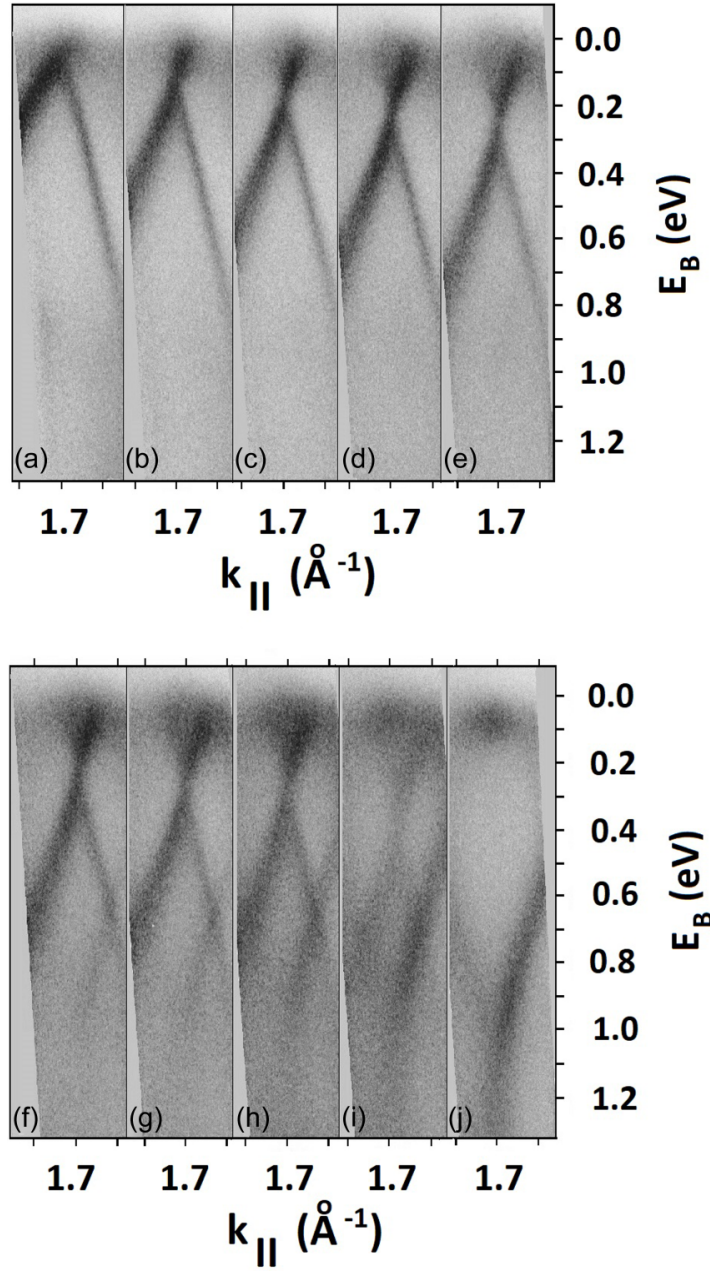

FIG. 1. ARPES spectra of $\mathrm{Gr} / \mathrm{Ir}(111)$ around the $K$ point along $\Gamma-K-M$ as a function of $\mathrm{Na}$ deposition time (see the text).

\section{RESULTS AND DISCUSSION}

The high quality of prepared graphene was, prior to sodium exposure, judged by the spectral linewidth of graphene $\pi$ bands (see Fig. 4 in Ref. [47]). The current through the Na dispenser was adjusted to ensure a low deposition rate in order to capture any possible change in the graphene band structure during $\mathrm{Na}$ adsorption/intercalation. Figure 1 shows the dispersion of graphene $\pi$ bands in a narrow momentum window around the $K$ point as a function of $\mathrm{Na}$ deposition time. As expected, due to electron doping, Na induces a shift of the Dirac point to higher binding energy which saturates after $20 \mathrm{~min}$ at an energy of around $0.3 \mathrm{eV}$ [see Fig. 1(f)]. Further deposition (29 min) does not affect the Dirac point or the shape of the Dirac cone. However, more Na deposition does induce changes in the whole spectrum in the form of an additional graphene $\pi$ band [see Fig. 1(g)]. As the exposure time increases, a pattern of the second Dirac cone becomes more discernible, while the intensity of the first Dirac cone is eventually completely reduced. The spectrum after $120 \mathrm{~min}$ of $\mathrm{Na}$ exposure [Fig. 1(j)] exhibits graphene $\pi$ bands with the Dirac point around $1.2 \mathrm{eV}$. There also appears to be some intensity at the Fermi level which is associated with the iridium surface state [47].

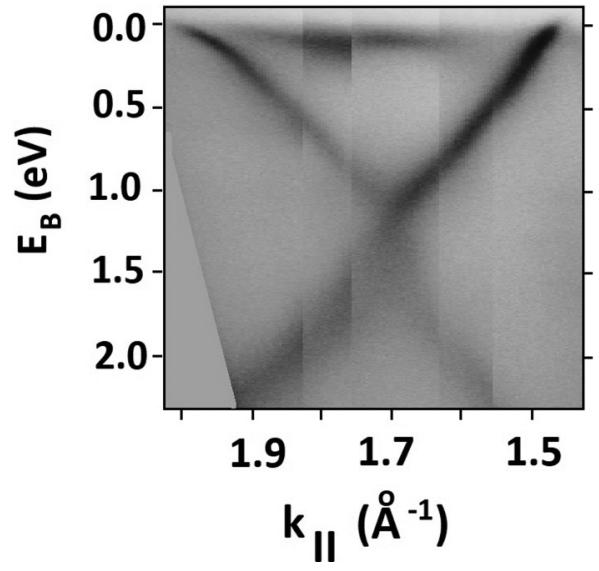

FIG. 2. ARPES spectra of $\operatorname{Gr} / \operatorname{Ir}(111)$ around the $K$ point along $\Gamma-K-M$ for the Na saturation coverage.

When the new Dirac cone emerges, further exposure to $\mathrm{Na}$ induces a steady shift of the Dirac point to higher binding energies such that for the $\mathrm{Na}$ saturation coverage the Dirac point reaches an energy of around $1.3 \mathrm{eV}$ (see Fig. 2), which is somewhat smaller than observed previously [7]. At the saturation coverage, we could not observe any Na-induced superstructure. This result is consistent with the observation of Papagno et al. [7], who were not able to detect any Na-induced superstructure in their STM images either. They concluded that Na saturation coverage was most likely consistent with the $(2 \times 2)$ periodicity [7] corresponding to $\Theta_{N a}=0.125$ with respect to graphene. ARPES maps shown in Figs. 1(h) and 1(i) are very similar to the spectrum observed for $\mathrm{Cs} / \mathrm{Gr} / \mathrm{Ir}(111)$, which showed the coexistence of adsorbed and intercalated phases of Cs [35]. Based on this simple analogy, we can put forward a hypothesis that the spectra with the Dirac point at 0.3 and $1.3 \mathrm{eV}$ below the Fermi level correspond to the adsorbed and intercalated phases, respectively. This assumption is clearly in contradiction to the conclusion of Papagno et al. [7], who found that the band structure of graphene saturated by $\mathrm{Na}$ with the Dirac cone at $1.5 \mathrm{eV}$ was associated with the $\mathrm{Na}$ atoms adsorbed on top of graphene. In order to resolve whether our ARPES spectrum of strongly $n$ doped graphene corresponds to either adsorbed or intercalated graphene, we performed an oxygen exposure experiment. Matyaba et al. [2] unambiguously demonstrated that intercalated $\mathrm{Na}$ is protected from oxidation by graphene so that extended exposure to oxygen does not affect the ARPES spectrum. However, when $\mathrm{Na}$ atoms are adsorbed on top of graphene the exposure to oxygen dramatically changes the valence-band spectrum around the $K$ point. Instead of the graphene $\pi$ bands, two nondispersing states related to sodium oxides emerged (see Fig. S3 in Ref. [2]). As Fig. 3 suggests, after exposure of $\mathrm{Gr} / \mathrm{Na} / \mathrm{Ir}(111)$ to $100 \mathrm{~L}$ of oxygen, no change in the valence-band structure around the $K$ point was observed either. Figure 3 shows a portion of the graphene spectrum around $k_{F} \approx 1.45 \AA^{-1}$. Any shift of the Dirac point towards the Fermi level (due to the oxidation of the Na layer) would lead to, as a consequence, a decrease in the Fermi surface, i.e., a shift of $k_{F}$ closer to the $K$ point. The change in $k_{F}$ is negligibly small, indicating that $\mathrm{Na}$ was protected by the 


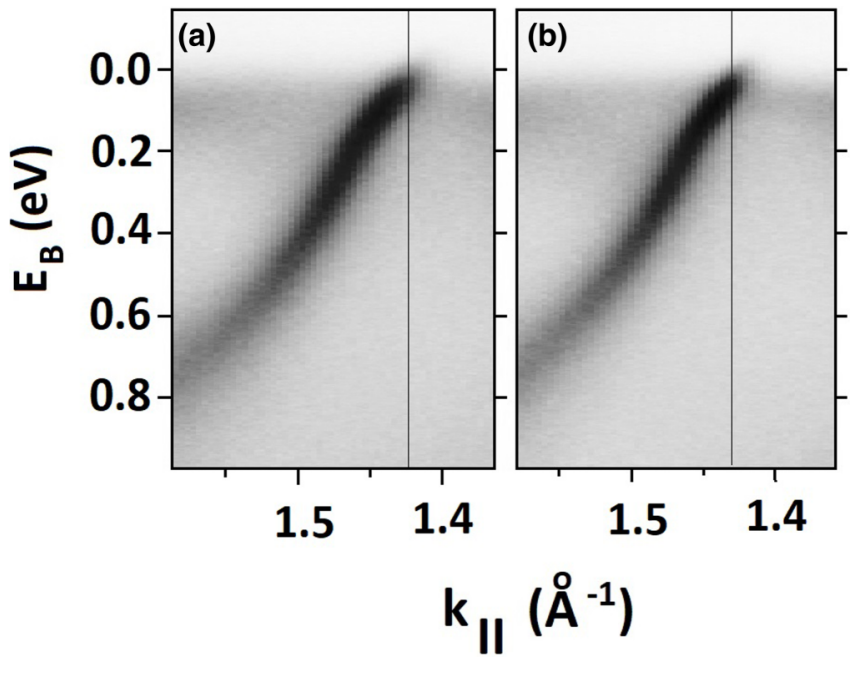

FIG. 3. ARPES spectrum of the $\Gamma-K-M$ branch of $\mathrm{Gr} / \mathrm{Na} / \mathrm{Ir}(111)$ (a) prior to and (b) after exposure to $100 \mathrm{~L}$ of oxygen.

graphene mesh. From these results, we draw the conclusion that the ARPES spectrum shown in Fig. 2 corresponds to $\mathrm{Na}$ intercalated between graphene and the Ir surface.

After completion of the Na-intercalated layer any attempt to adsorb more $\mathrm{Na}$ on top of graphene failed as additional exposure to $\mathrm{Na}$ introduced no change in the valence-band spectrum. However, we did see a noticeable change in the spectrum if the $\mathrm{Gr} / \mathrm{Na} / \mathrm{Ir}(111)$ sample was exposed to $\mathrm{Li}$ instead. In the following, we shall demonstrate that the deposited $\mathrm{Li}$ is not intercalated but adsorbed on top of the $\mathrm{Gr} / \mathrm{Na} / \mathrm{Ir}(111)$ system. The exposure to Li induces a significant shift in the Dirac point to even higher binding energies (from 1.3 to $1.7 \mathrm{eV}$ ). This is one of the largest binding energies measured for any doped graphene system [see Fig. 4(a)]. In addition to the shift of the Dirac point, there is a substantial shift of the Ir surface state. The same effect was recently reported for graphene on $\operatorname{Ir}(111)$ intercalated by only $\mathrm{Li}$ [38]. Instead of quenching the surface state, as some other intercalated atoms do [61], Li atoms placed between graphene and the $\operatorname{Ir}(111)$ surface only increase their binding energy without affecting the coherence around the $K$ point [38]. However, in this case the surface state shift is not induced by intercalated $\mathrm{Li}$ but rather by adsorbed $\mathrm{Li}$, although the spectroscopic appearance and the binding energy (around $0.45 \mathrm{eV}$ ) are the same. The oxygen test was again applied in order to test the Li position with respect to graphene.

As can be seen in Fig. 4(b), the exposure of the $\mathrm{Li} / \mathrm{Gr} / \mathrm{Na} / \mathrm{Ir}(111)$ system induces an almost rigid shift of all the bands towards the Fermi level and, consequently, the decrease of $k_{F}$ from 1.61 to $1.52 \AA^{-1}$. This shift clearly suggests that oxygen has retrieved electrons that have been transferred from Li to graphene, thus reducing the $n$ doping of the $\mathrm{Gr} / \mathrm{Na} / \mathrm{Ir}(111)$ system. We are confident that deposited $\mathrm{Li}$ is in the adsorbed state and not in the intercalated state because the previous experiments of $\mathrm{Gr} / \mathrm{Li} / \mathrm{Ir}(111)$ exposure to oxygen showed no change in the valence-band spectrum whatsoever. This raises the question of why Li adsorbs on the $\mathrm{Gr} / \mathrm{Na} / \mathrm{Ir}(111)$ system and Na does not. Recently, it was unambiguously demonstrated

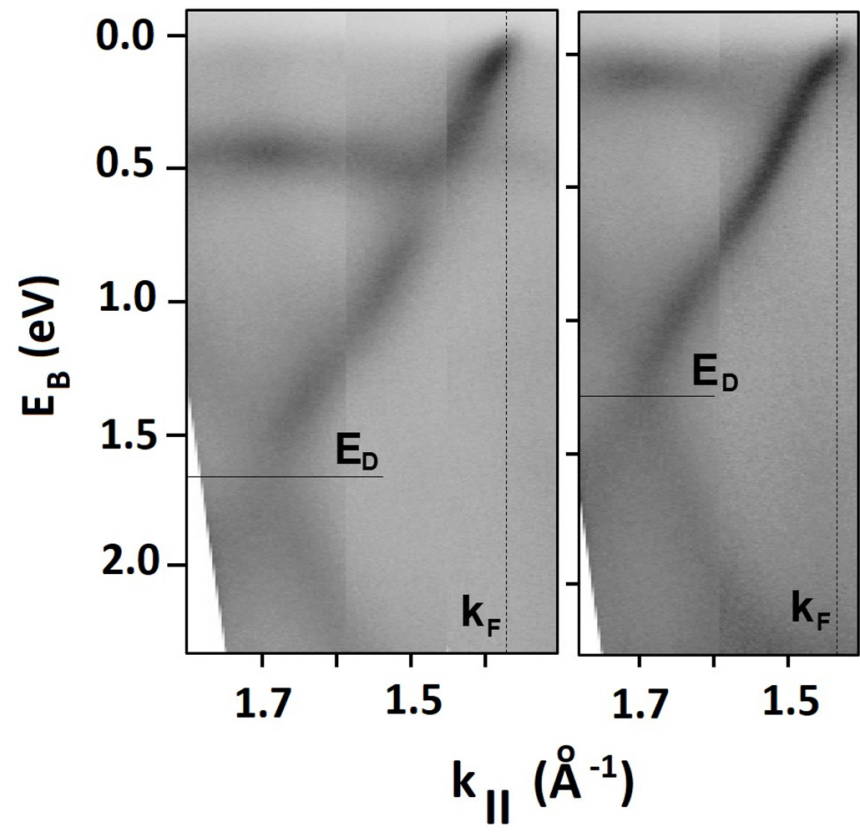

FIG. 4. ARPES spectrum of $\mathrm{Li} / \mathrm{Gr} / \mathrm{Na} / \mathrm{Ir}(111)$ along $\Gamma-K-M$ before (left) and after (right) oxygen exposure (100 L).

that $\mathrm{Cs}$ and Eu do not adsorb at RT on graphene when it is intercalated by $\mathrm{Cs}$ and Eu, respectively [62]. The STM data were corroborated by DFT calculations suggesting a strong reduction of the $\mathrm{Cs}(\mathrm{Eu})$ adsorption energy on the order of $1 \mathrm{eV}$ when graphene is intercalated by $\mathrm{Cs}(\mathrm{Eu})$. As we shall demonstrate shortly, the fact that $\mathrm{Na}$ does not adsorb on $\mathrm{Gr} / \mathrm{Na} / \mathrm{Ir}(111)$ while Li does can be explained in the same fashion, i.e., by the difference in their $\mathrm{Na}$ intercalation-induced adsorption energies. However, the reduction of adsorption energy on intercalated graphene might not always be big enough to prevent the coexistence of intercalated and adsorbed states at RT as in the case of $\mathrm{K} / \mathrm{Gr} / \mathrm{K} / \mathrm{Ir}(111)$ [40]. As the experiment showed, elevated temperatures (above $360 \mathrm{~K}$ ) are required in order to suppress $\mathrm{K}$ adsorption on $\mathrm{Gr} / \mathrm{K} / \mathrm{Ir}(111)$ [40].

Before discussing the nature of $\mathrm{Na}$ and $\mathrm{Li}$ bonding to intercalated graphene, we would like to address the observation that $\mathrm{Na}$ intercalation does not induce a shift of the Ir surface state below the Fermi level, while Li does [38]. As Fig. 3(b) in Ref. [38] clearly shows, initially, it takes a significant number of $\mathrm{Li}$ atoms (i.e., the charge transferred to graphene and the Ir surface) to make a minor shift of the surface state, not larger than $50 \mathrm{meV}$. It should be noticed that this small shift corresponds to a reduction in the binding energy of the Dirac point as large as $1.3 \mathrm{eV}$, which is exactly the energy shift observed for graphene intercalated by Na (see Fig. 2). The conclusion is as follows: due to the larger size of $\mathrm{Na}$ atoms compared to $\mathrm{Li}$, the saturation coverage of intercalated $\mathrm{Na}$ is, in absolute terms, smaller than the saturation coverage of Li. As a consequence, there is a lack of charge transferred to Ir to facilitate the shift of the surface state below the Fermi level. This finding is consistent with the notion that the $\mathrm{Na}$ saturation coverage corresponds to a $(2 \times 2)$ superstructure, i.e., $\Theta_{N a}=0.125$. However, when additional charge is provided by adsorbed $\mathrm{Li}$ atoms, the surface state indeed 


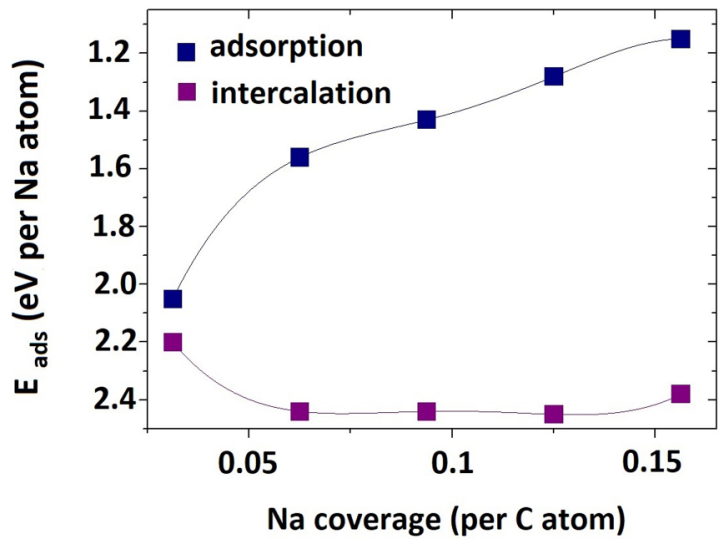

FIG. 5. Calculated adsorption vs intercalation energies for increasing Na coverage. Calculated energy equals the total energy of the system divided by the number of adsorbed/intercalated $\mathrm{Na}$ atoms.

exhibits a clear departure from the Fermi level. Moreover, due to their larger size $\mathrm{Na}$ atoms tend to create a metallic layer at much lower coverages than $\mathrm{Li}$ atoms, resulting in the appearance of two-dimensional electron-gas states which can further accommodate $\mathrm{Na}$ electrons. This effect significantly reduces the donation of $\mathrm{Na}$ electrons to graphene or iridium.

Next, we provide theoretical support for the observed mechanism of $\mathrm{Na}$ adsorption/intercalation of graphene on $\operatorname{Ir}(111)$. As Fig. 5 shows, initial adsorption of $\mathrm{Na}$ on $\mathrm{Gr} / \mathrm{Ir}(111)$ is just slightly less energetically favorable than intercalation. The calculation of Jin et al. [63] also shows that adsorption of two $\mathrm{Na}$ atoms on the same side of graphene is energetically less favorable than on opposite sides of the graphene layer (one $\mathrm{Na}$ atom on each side), thereby supporting the picture of the intercalation process. However, being initially adsorbed, a $\mathrm{Na}$ atom does not experience a particularly strong driving force to change its state from adsorption to intercalation. Yet, as the concentration of adsorbed $\mathrm{Na}$ increases, the intercalation becomes significantly energetically more favorable (by around $0.9 \mathrm{eV}$ ). At $\Theta_{N a}=0.125$ the energy difference in favor of intercalation rises to $1.15 \mathrm{eV}$. Therefore, the increasing coverage of adsorbed $\mathrm{Na}$ exerts "pressure" on $\mathrm{Na}$ atoms to take the position of higher binding energy, i.e., of the intercalated state. As has been compellingly demonstrated in the case of Cs intercalation of $\mathrm{Gr} / \operatorname{Ir}(111)$, Cs coverage has to reach a value of $0.1 \mathrm{ML}$ (corresponding to $\Theta_{C s} \approx 0.03$ per carbon atom) before the channel for intercalation is opened. A similar pattern of coexistence of the AM adsorbed and intercalated states that precedes fully intercalated graphene was recently reported for $\mathrm{K} / \mathrm{Gr} / \mathrm{Ir}(111)$ [40]. Judging from the coverage dependence of $\mathrm{Na}$ adsorption vs intercalation energy (see Fig. 5), we estimate that the coverage $\Theta_{N a} \approx 0.05$ is required in this case before intercalation sets in. Notice also that with increasing $\mathrm{Na}$ coverage the intercalation energy remains constant while at the same time the adsorption energy experiences a substantial decrease, making the process of intercalation at higher $\mathrm{Na}$ coverages even more likely. This widening energy gap between adsorption and intercalated state supports our view that at RT an increased supply of $\mathrm{Na}$ atoms on graphene accelerates the intercalation process. It is obvious that the mobility of adsorbed AM on graphene should play

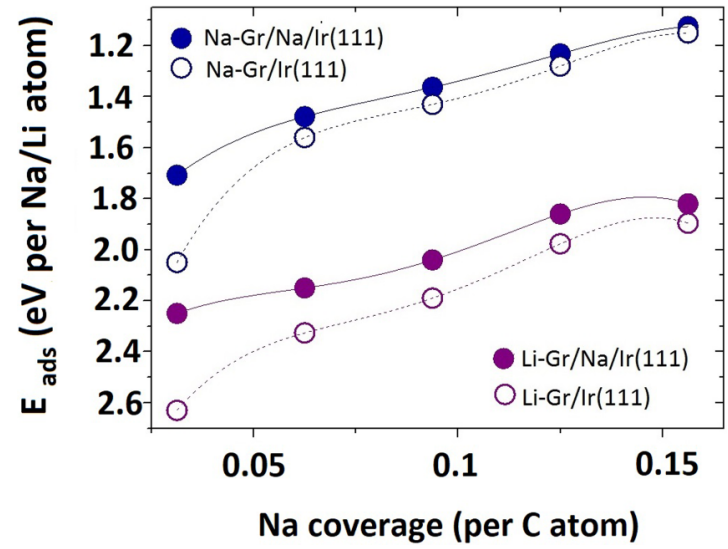

FIG. 6. Calculated adsorption energies of $\mathrm{Na}$ and $\mathrm{Li}$ on $\mathrm{Gr} / \mathrm{Na} / \mathrm{Ir}(111)$ for increasing $\mathrm{Na} / \mathrm{Li}$ coverage and saturating $\mathrm{Na}$ concentration in the intercalated state. Also shown for comparison are the coverage dependences of adsorption energies of $\mathrm{Li}$ and $\mathrm{Na}$ on $\mathrm{Gr} / \mathrm{Ir}(111)$. The lines are to guide the eye.

an important role in the kinetics of the intercalation process. A repulsive force between induced dipoles of adsorbed AM facilitates the motion of adatoms across graphene in finding their path to underneath graphene [35]. However, since this is an activated process, it is not surprising that the surface temperature can have a decisive impact on the mobility and, ultimately, on the intercalation of AM adatoms. It has been shown that adsorption of $\mathrm{K}$ on $\mathrm{Gr} / \mathrm{Ir}(111)$ at $80 \mathrm{~K}$ freezes out the intercalation process entirely, and only the annealing at $\mathrm{RT}$ and higher (up to $360 \mathrm{~K}$ ) provides $\mathrm{K}$ adatoms with large enough mobility to promote intercalation [40].

Figure 6 shows the concentration dependence of the $\mathrm{Na}(\mathrm{Li})$ adsorption energy on graphene that was previously intercalated by $\mathrm{Na}$. Note that the adsorption energy of an isolated $\mathrm{Na}$ atom on intercalated graphene $\left[E_{\text {ads }}(\mathrm{I})=1.75 \mathrm{eV}\right]$ is reduced by $0.3 \mathrm{eV}$ with respect to nonintercalated graphene $\left[E_{\mathrm{ads}}(\mathrm{NI})=\right.$ $2.05 \mathrm{eV}]$.We observe that a similar reduction of adsorption energy on intercalated graphene is also experienced by an isolated $\mathrm{Li}$ atom. The decrease in adsorption energy upon graphene intercalation is not as large as for Cs or Eu but is obviously large enough to have a similar impact on the adsorption kinetics [62] of $\mathrm{Na}$ and Li. We see from Fig. 6 that for all $\mathrm{Na}$ and $\mathrm{Li}$ concentrations on $\mathrm{Gr} / \mathrm{Na} / \mathrm{Ir}(111)$ lithium atoms exhibit a noticeably larger adsorption energy. Previous theoretical calculations of $\mathrm{Li}$ and $\mathrm{Na}$ adsorption energies consistently gave significantly larger values for $\mathrm{Li}$ relative to $\mathrm{Na}$ in the case of unsupported graphene as well as for $\mathrm{Gr} / \mathrm{Au} / \mathrm{Ni}(111)$ (see Table II in Ref. [36]). At this point, it is appropriate to address the nature of the difference of the strength of bonding of $\mathrm{Li}$ and $\mathrm{Na}$ to graphene. Namely, since $\mathrm{Na}$ is a more reactive alkali atom than $\mathrm{Li}$, it should benefit more from charge donation to graphene in ionic bond formation. From that point of view, Na should bond more strongly to graphene than Li. However, due to the small size of the $\mathrm{Li}$ atom the charge it donates stays within the Li atomic potential, making the bond with graphene more covalentlike, from which $\mathrm{Li}$ benefits by creating a stronger bond. In their analysis of $\mathrm{Li}^{+}$ and $\mathrm{Na}^{+}$interaction with benzene complexes Tsuzuki et al. [64] emphasized the significance of the so-called induction 

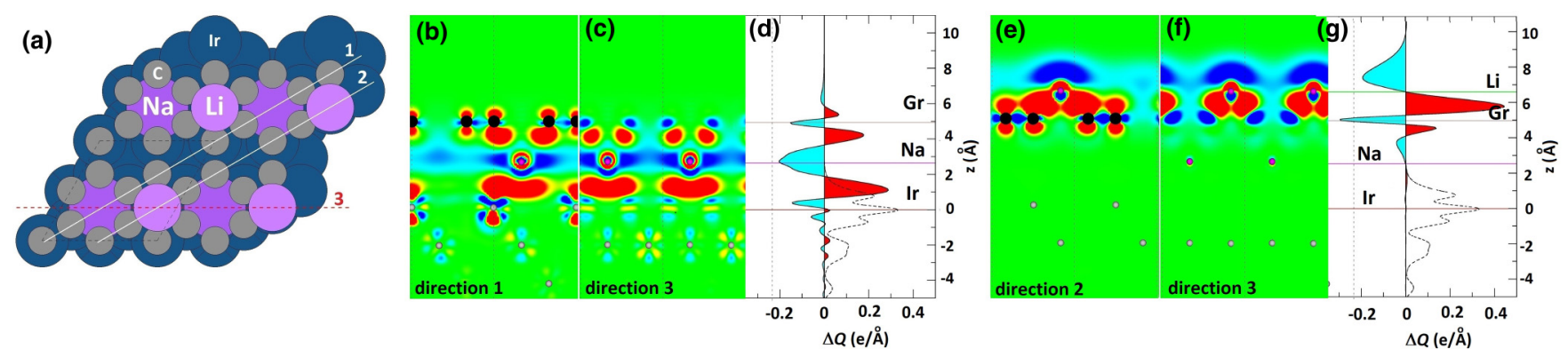

FIG. 7. (a) Structural model of $\mathrm{Li} / \mathrm{Gr} / \mathrm{Na} / \mathrm{Ir}(111)$ and $\mathrm{Gr} / \mathrm{Na} / \mathrm{Ir}(111)$ systems indicating three directions along which the calculated chargetransfer density maps are shown in (b) Gr/Na/Ir(111) along direction 1, (c) Gr/Na/Ir(111) along direction 3, (e) Li/Gr/Na/Ir(111) along direction 2, and (f) $\mathrm{Li} / \mathrm{Gr} / \mathrm{Na} / \mathrm{Ir}(111)$ along direction 3. (d) and (g) show the corresponding charge rearrangement along the $z$ direction. (d) Charge transfer calculated as the charge difference $Q(\mathrm{Gr} / \mathrm{Na} / \mathrm{Ir})-[Q(\mathrm{Gr} / \mathrm{Ir})+Q(\mathrm{Na})]$. (g) Charge transfer calculated as charge difference $Q(\mathrm{Ir} / \mathrm{Na} / \mathrm{Gr} / \mathrm{Li})-[Q(\mathrm{Ir} / \mathrm{Na} / \mathrm{Gr})+Q(\mathrm{Li})]$.

energy which is the interaction energy between the polarizable sites of the benzene complexes and the electric field produced by $\mathrm{Li}^{+}$and $\mathrm{Na}^{+}$. Due to the $R^{-4}$ dependence ( $R$ is the distance from the AM cation) of the induction energy, its contribution to Li bonding is more than two times larger than that to $\mathrm{Na}$, making the total bonding energy of $\mathrm{Li}^{+} /$benzene larger than that of $\mathrm{Na}^{+}$/benzene [64].

We have also explored within the DFT framework the charge transferred to graphene and iridium upon $\mathrm{Na}$ intercalation and additional $\mathrm{Li}$ adsorption. Figure 7(a) shows a structural model of a $\mathrm{Li}(2 \times 2)+\mathrm{Gr} / \mathrm{Na}(2 \times 2) / \operatorname{Ir}(111)$ superstructure with three directions along which the chargetransfer density maps have been calculated. Figures 7(b) and 7(c) show how the charge released by intercalated $\mathrm{Na}$ redistributes to $\mathrm{Gr}$ and Ir surfaces along directions 1 and 3, respectively. Figure 7(d) displays the corresponding integrated charge rearrangement along the $z$ direction. We see that a significant amount of charge has been transferred from the $\mathrm{Na}$ atoms to graphene and Ir. An additional analysis has shown that, likewise, in the case of Li intercalation of graphene [38], the charge transferred from $\mathrm{Na}$ to graphene and the Ir surface is almost additive in nature. In other words, the total charge transferred to $\mathrm{Gr}$ and Ir through the intercalation is nearly equal to the charge transferred independently from $\mathrm{Na}$ to $\mathrm{Gr}$ (freestanding Gr) and $\operatorname{Ir}(111)$. Additional interesting information arises from the charge-transfer density maps which illustrate how the electronic charge is transferred to Gr and Ir not only from the areas around $\mathrm{Na}$ atoms but also from between them. This clearly indicates that at such distances $\mathrm{Na}$ atoms are already forming a metallic layer. Figures 7(e) and 7(f) show the calculated charge-transfer density maps of the $\mathrm{Li} / \mathrm{Gr} / \mathrm{Na} / \mathrm{Ir}(111)$ systems along directions 2 and 3, respectively. Figure 7(g) displays the corresponding charge rearrangement along the $z$ direction. It is obvious that most of the charge released by $\mathrm{Li}$ goes to Gr, while the effect on the Ir surface appears to be reflected merely through the charge redistribution. However, the experimentally observed shift of the Ir surface state upon $\mathrm{Li}$ adsorption indicates that there has been some potential change at the interface which induces a shift of the surface state to higher binding energies. Li adsorption charge-transfer density maps [Figs. 7(e) and 7(f)] suggest that apart from the reduction of charge around $\mathrm{Li}$ atoms, there is a charge density decrease in between, indicating that for a $(2 \times 2)$ superstructure $\mathrm{Li}$ creates a metallic layer as well. This is consistent with DFT calculations by Jin et al. [63], who showed that despite the fact that $\mathrm{Na}$ and $\mathrm{Li}$ exhibit a crossover from an isolated $\mathrm{AM}$ to a metallic layer at different coverages, $\mathrm{Na}$ and $\mathrm{Li}$ should form a metallic layer at the concentration corresponding to the $(2 \times 2)$ superstructure.

To summarize, we have demonstrated that $\mathrm{Na}$ initially adsorbs on $\mathrm{Gr} / \mathrm{Ir}(111)$ at RT but intercalates with continuous exposure. These two states reflect the different electron doping of graphene which is observed through different shifts of the Dirac cone; adsorbed $\mathrm{Na}$ induces a shift of the Dirac point to $0.3 \mathrm{eV}$, while the signature of the fully intercalated $\mathrm{Na}$ is the Dirac point at $1.3 \mathrm{eV}$ below the Fermi level. DFT calculations support this scenario, showing that the adsorption of $\mathrm{Na}$ is the energetically favorable state relative to intercalation only at low $\mathrm{Na}$ concentrations. The increase in $\mathrm{Na}$ coverage on the graphene surface significantly reduces adsorption with respect to intercalation energy, building up the pressure for intercalation. This response indicates that the corresponding intercalation kinetics is very similar to that of Cs [35], most likely of $\mathrm{K}$ on $\mathrm{Gr} / \mathrm{Ir}(111)$ [40]. We have also demonstrated that once $\mathrm{Na}$ has been intercalated, no additional $\mathrm{Na}$ can be adsorbed on graphene at RT, which is explained in terms of reduction of $\mathrm{Na}$ adsorption energy upon $\mathrm{Na}$ intercalation. However, we have shown that Li does adsorb on $\mathrm{Gr} / \mathrm{Na} / \mathrm{Ir}(111)$ as $\mathrm{Li}$ experiences a stronger bonding to graphene than $\mathrm{Na} . \mathrm{Li}$ adsorption induces a substantial shift of the Ir surface state to higher binding energy, similar to $\mathrm{Gr} / \mathrm{Li} / \mathrm{Ir}(111)$.

\section{ACKNOWLEDGMENTS}

We thank B. Gumhalter for useful discussions. P.P. acknowledges the financial support of the Croatian Science Foundation (Grant No. IP-11-2013-2727). P.L. was supported by the Unity through Knowledge Fund, Contract No. 22/15, and Horizon 2020 CSA Twinning Project No. 692194, RBI-T-WINNING. 
[1] C. Coletti, C. Riedl, D. S. Lee, B. Krauss, L. Patthey, K. Von Klitzing, J. H. Smet, and U. Starke, Charge neutrality and bandgap tuning of epitaxial graphene on $\mathrm{SiC}$ by molecular doping, Phys. Rev. B 81, 235401 (2010).

[2] P. Matyba, A. Carr, C. Chen, D. L. Miller, G. Peng, S. Mathias, M. Mavrikakis, D. S. Dessau, M. W. Keller, H. C. Kapteyn, and M. Murnane, Controlling the electronic structure of graphene using surface-adsorbate interactions, Phys. Rev. B 92, 041407 (2015).

[3] A. Varykhalov, M. R. Scholz, T. K. Kim, and O. Rader, Effect of noble-metal contacts on doping and band gap of graphene, Phys. Rev. B 82, 121101 (2010).

[4] A. Grüneis and D. V. Vyalikh, Tunable hybridization between electronic states of graphene and a metal surface, Phys. Rev. B 77, 193401 (2008).

[5] D. C. Elias, R. R. Nair, T. M. G. Mohiuddin, S. V Morozov, P. Blake, M. P. Halsall, A. C. Ferrari, D. W. Boukhvalov, M. I. Katsnelson, A. K. Geim, and K. S. Novoselov, Control of graphene's properties by reversible hydrogenation: Evidence for graphane, Science 323, 610 (2009).

[6] D. Usachov, O. Vilkov, A. Grüneis, D. Haberer, A. Fedorov, V. K. Adamchuk, A. B. Preobrajenski, P. Dudin, A. Barinov, M. Oehzelt, C. Laubschat, and D. V. Vyalikh, Nitrogen-doped graphene: Efficient growth, structure, and electronic properties, Nano Lett. 11, 5401 (2011).

[7] M. Papagno, S. Rusponi, P. M. Sheverdyaeva, S. Vlaic, M. Etzkorn, D. Pacilé, P. Moras, C. Carbone, and H. Brune, Large band gap opening between graphene Dirac cones induced by $\mathrm{Na}$ adsorption onto an Ir superlattice, ACS Nano 6, 199 (2012).

[8] J. L. McChesney, A. Bostwick, T. Ohta, T. Seyller, K. Horn, J. González, and E. Rotenberg, Extended Van Hove Singularity and Superconducting Instability in Doped Graphene, Phys. Rev. Lett. 104, 136803 (2010).

[9] A. Bostwick, F. Speck, T. Seyller, K. Horn, M. Polini, R. Asgari, A. H. MacDonald, and E. Rotenberg, Observation of plasmarons in quasi-freestanding doped graphene, Science 328, 999 (2010).

[10] A. Politano and G. Chiarello, Alkali-induced hydrogenation of epitaxial graphene by water splitting at $100 \mathrm{~K}$, J. Chem. Phys. 138, 044703 (2013).

[11] S. S. Harilal, J. P. Allain, A. Hassanein, M. R. Hendricks, and M. Nieto-Perez, Reactivity of lithium exposed graphite surface, Appl. Surf. Sci. 255, 8539 (2009).

[12] B. Heim, C. N. Taylor, and J. P. Allain, Tuning the electronic and chemical surface properties of graphane combining alkali deposition and low-energy ion irradiation, in Nanotechnol. 2010 Adv. Mater. CNTs, Part. Film. Compos. - 2010 NSTI Nanotechnol. Conf. Expo, NSTI-Nanotech 2010, June 21, 2010 - June 24, 2010 (unpublished).

[13] T. Kihlgren, T. Balasubramanian, L. Walldén, and R. Yakimova, $\mathrm{K} /$ graphite: Uniform energy shifts of graphite valence states, Surf. Sci. 600, 1160 (2006).

[14] A. Bostwick, T. Ohta, T. Seyller, K. Horn, and E. Rotenberg, Quasiparticle dynamics in graphene, Nat. Phys. 3, 36 (2007).

[15] C. G. Hwang, S. Y. Shin, S. M. Choi, N. D. Kim, S. H. Uhm, H. S. Kim, C. C. Hwang, D. Y. Noh, S. H. Jhi, and J. W. Chung, Stability of graphene band structures against an external periodic perturbation: Na on graphene, Phys. Rev. B 79, 115439 (2009).

[16] V. Fedorov, N. I. Verbitskiy, D. Haberer, C. Struzzi, L. Petaccia, D. Usachov, O. Y. Vilkov, D. V Vyalikh, J. Fink,
M. Knupfer, B. Büchner, and A. Grüneis, Observation of a universal donor-dependent vibrational mode in graphene, Nat. Commun. 5, 3257 (2014).

[17] T. Valla, J. Camacho, Z. H. Pan, A. V. Fedorov, A. C. Walters, C. A. Howard, and M. Ellerby, Anisotropic Electron-Phonon Coupling and Dynamical Nesting on the Graphene Sheets in Superconducting $\mathrm{CaC}_{6}$ Using Angle-Resolved Photoemission Spectroscopy, Phys. Rev. Lett. 102, 107007 (2009).

[18] A. Cupolillo, N. Ligato, and L. S. Caputi, Plasmon dispersion in quasi-freestanding graphene on Ni(111), Appl. Phys. Lett. 102, 111609 (2013).

[19] I. Pletikosić, M. Kralj, M. Milun, and P. Pervan, Finding the bare band: Electron coupling to two phonon modes in potassium-doped graphene on $\operatorname{Ir}(111)$, Phys. Rev. B 85, 155447 (2012).

[20] B. M. Ludbrook, G. Levy, P. Nigge, M. Zonno, M. Schneider, D. J. Dvorak, C. N. Veenstra, S. Zhdanovich, D. Wong, P. Dosanjh, C. Straßer, A. Stöhr, S. Forti, C. R. Ast, U. Starke, and A. Damascelli, Evidence for superconductivity in Li-decorated monolayer graphene, Proc. Natl. Acad. Sci. USA 112, 11795 (2015).

[21] G. Profeta, M. Calandra, and F. Mauri, Phonon-mediated superconductivity in graphene by lithium deposition, Nat. Phys. 8, 131 (2012).

[22] A. F. Hebard, M. J. Rosseinsky, R. C. Haddon, D. W. Murphy, S. H. Glarum, T. T. M. Palstra, A. P. Ramirez, and A. R. Kortan, Superconductivity at $18 \mathrm{~K}$ in potassium-doped $\mathrm{C}_{60}$, Nature (London) 350, 600 (1991).

[23] E. Voloshina and Y. Dedkov, Graphene on metallic surfaces: Problems and perspectives, Phys. Chem. Chem. Phys. 14, 13502 (2012).

[24] D. Eom, D. Prezzi, K. T. Rim, H. Zhou, M. Lefenfeld, S. Xiao, C. Nuckolls, M. S. Hybertsen, T. F. Heinz, and G. W. Flynn, Structure and electronic properties of graphene nanoislands on Co(0001), Nano Lett. 9, 2844 (2009).

[25] P. W. Sutter, J.-I. Flege, and E. A. Sutter, Epitaxial graphene on ruthenium, Nat. Mater. 7, 406 (2008).

[26] Y. S. Park, J. H. Park, H. N. Hwang, T. S. Laishram, K. S. Kim, M. H. Kang, and C. C. Hwang, Quasi-Free-Standing Graphene Monolayer on a Ni Crystal through Spontaneous Na Intercalation, Phys. Rev. X 4, 031016 (2014).

[27] M. Alattas and U. Schwingenschlögl, Quasi-freestanding graphene on $\mathrm{Ni}(111)$ by Cs intercalation, Sci. Rep. 6, 26753 (2016).

[28] N. Ligato, A. Cupolillo, and L. S. Caputi, Study of the intercalation of graphene on $\mathrm{Ni}(111)$ with $\mathrm{Cs}$ atoms: Towards the quasi-free graphene, Thin Solid Films 543, 59 (2013).

[29] M. S. Dresselhaus and G. Dresselhaus, Intercalation compounds of graphite, Adv. Phys. 51, 1 (1981).

[30] M. Caragiu and S. Finberg, Alkali metal adsorption on graphite: A review, J. Phys. Condens. Matter 17, R995 (2005).

[31] K. M. Hock and R. E. Palmer, Temperature dependent behaviour in the adsorption of submonolayer potassium on graphite, Surf. Sci. 284, 349 (1993).

[32] G. Yoon, H. Kim, I. Park, and K. Kang, Conditions for reversible $\mathrm{Na}$ intercalation in graphite: Theoretical studies on the interplay among guest ions, solvent, and graphite host, Adv. Energy Mater. 7, 1601519 (2017)

[33] J. S. Bunch, S. S. Verbridge, J. S. Alden, A. M. Van Der Zande, J. M. Parpia, H. G. Craighead, and P. L. McEuen, Impermeable 
atomic membranes from graphene sheets, Nano Lett. 8, 2458 (2008).

[34] D. W. Boukhvalov and C. Virojanadara, Penetration of alkali atoms throughout a graphene membrane: theoretical modeling, Nanoscale 4, 1749 (2012).

[35] M. Petrović, I. Šrut Rakić, S. Runte, C. Busse, J. T. Sadowski, P. Lazić, I. Pletikosić, Z.-H. Pan, M. Milun, P. Pervan, N. Atodiresei, R. Brako, D. Šokčević, T. Valla, T. Michely, and M. Kralj, The mechanism of caesium intercalation of graphene, Nat. Commun. 4, 2772 (2013).

[36] C. S. Praveen, S. Piccinin, and S. Fabris, Adsorption of alkali adatoms on graphene supported by the $\mathrm{Au} / \mathrm{Ni}(111)$ surface, Phys. Rev. B 92, 075403 (2015).

[37] J. Halle, N. Néel, and J. Kröger, Filling the gap: Li-intercalated graphene on Ir(111), J. Phys. Chem. C 120, 5067 (2016).

[38] P. Pervan, P. Lazić, M. Petrović, I. Šrut Rakić, I. Pletikosić, M. Kralj, M. Milun, and T. Valla, Li adsorption versus graphene intercalation on $\operatorname{Ir}(111)$ : From quenching to restoration of the $\mathrm{Ir}$ surface state, Phys. Rev. B 92, 245415 (2015).

[39] F. Bisti, G. Profeta, H. Vita, M. Donarelli, F. Perrozzi, P. M. Sheverdyaeva, P. Moras, K. Horn, and L. Ottaviano, Electronic and geometric structure of graphene/ $\mathrm{SiC}(0001)$ decoupled by lithium intercalation, Phys. Rev. B 91, 245411 (2015).

[40] C. Struzzi, C. S. Praveen, M. Scardamaglia, N. I. Verbitskiy, A. V. Fedorov, M. Weinl, M. Schreck, A. Grüneis, S. Piccinin, S. Fabris, and L. Petaccia, Controlled thermodynamics for tunable electron doping of graphene on $\operatorname{Ir}(111)$, Phys. Rev. B 94, 085427 (2016).

[41] M. Bianchi, E. D. L. Rienks, S. Lizzit, A. Baraldi, R. Balog, L. Hornekær, and P. Hofmann, Electron-phonon coupling in potassium-doped graphene: Angle-resolved photoemission spectroscopy, Phys. Rev. B 81, 041403 (2010).

[42] A. Nagashima, N. Tejima, and C. Oshima, Electronic states of the pristine and alkali-metal-intercalated monolayer graphite/Ni(111) systems, Phys. Rev. B 50, 17487 (1994).

[43] M. T. Johnson, H. I. Starnberg, and H. P. Hughes, Electronic structure of alkali metal overlayers on graphite, Surf. Sci. 178, 290 (1986).

[44] N. R. Gall, E. V. Rut'kov, and A. Ya. Tontegode, Two dimensional graphite films on metals and their intercalation, Int. J. Mod. Phys. 11, 1865 (1996).

[45] T. Langer, D. F. Förster, C. Busse, T. Michely, H. Pfnür, and C. Tegenkamp, Sheet plasmons in modulated graphene on $\operatorname{Ir}(111)$, New J. Phys. 13, 053006 (2011).

[46] I. Pletikosić, M. Kralj, D. Sokčević, R. Brako, P. Lazić, and P. Pervan, Photoemission and density functional theory study of $\operatorname{Ir}(111)$; energy band gap mapping, J. Phys.: Condens. Matter 22, 135006 (2010).

[47] M. Kralj, I. Pletikosić, M. Petrović, P. Pervan, M. Milun, A. T. N'Diaye, C. Busse, T. Michely, J. Fujii, and I. Vobornik,
Graphene on $\operatorname{Ir}(111)$ characterized by angle-resolved photoemission, Phys. Rev. B 84, 075427 (2011).

[48] K. M. Hock, J. C. Barnard, R. E. Palmer, and H. Ishida, Competing Routes for Charge Transfer in Co-Adsorption of $\mathrm{K}$ and $\mathrm{O}_{2}$ on Graphite, Phys. Rev. Lett. 71, 641 (1993).

[49] P. Hohenberg and W. Kohn, Inhomogeneous electron gas, Phys. Rev. 136, B864 (1964).

[50] W. Kohn and L. J. Sham, Self-consistent equations including exchange and correlation effects, Phys. Rev. 140, A1133 (1965).

[51] G. Kresse and J. Hafner, Ab initio molecular dynamics for liquid metals, Phys. Rev. B 47, 558 (1993).

[52] G. Kresse and J. Furthmüller, Efficient iterative schemes for $a b$ initio total-energy calculations using a plane-wave basis set, Phys. Rev. B 54, 11169 (1996).

[53] P. E. Blöchl, Projector augmented-wave method, Phys. Rev. B 50, 17953 (1994).

[54] G. Kresse and D. Joubert, From ultrasoft pseudopotentials to the projector augmented-wave method, Phys. Rev. B 59, 1758 (1999).

[55] M. Dion, H. Rydberg, E. Schröder, D. C. Langreth, and B. I. Lundqvist, Van der Waals Density Functional for General Geometries, Phys. Rev. Lett. 92, 246401 (2004).

[56] J. Klimeš, D. R. Bowler, and A. Michaelides, Van der Waals density functionals applied to solids, Phys. Rev. B 83, 195131 (2011).

[57] A. D. Becke, Density-functional exchange-energy approximation with correct asymptotic behavior, Phys. Rev. A 38, 3098 (1988).

[58] H. J. Monkhorst and J. D. Pack, Special points for Brillouin-zone integrations, Phys. Rev. B 13, 5188 (1976).

[59] G. Makov and M. C. Payne, Periodic boundary conditions in $a b$ initio calculations, Phys. Rev. B 51, 4014 (1995).

[60] J. Neugebauer and M. Scheffler, Adsorbate-substrate and adsorbate-adsorbate interactions of $\mathrm{Na}$ and $\mathrm{K}$ adlayers on Al(111), Phys. Rev. B 46, 16067 (1992).

[61] S. Ulstrup, M. Andersen, M. Bianchi, L. Barreto, B. Hammer, L. Hornekær, and P. Hofmann, Sequential oxygen and alkali intercalation of epitaxial graphene on $\operatorname{Ir}(111)$ : Enhanced manybody effects and formation of pn-interfaces, 2D Mater. 1, 025002 (2014).

[62] S. Schumacher, T. O. Wehling, P. Lazic, S. Runte, D. F. Forster, C. Busse, M. Petrovic, M. Kralj, S. Blucollectivegel, N. Atodiresei, V. Caciuc, and T. Michely, The backside of graphene: Manipulating adsorption by intercalation, Nano Lett. 13, 5013 (2013).

[63] K.-H. Jin, S.-M. Choi, and S.-H. Jhi, Crossover in the adsorption properties of alkali metals on graphene, Phys. Rev. B 82, 033414 (2010).

[64] S. Tsuzuki, M. Yoshida, T. Uchimaru, and M. Mikami, The origin of the cation/ $\pi$ interaction: The significant importance of the induction in $\mathrm{Li}^{+}$and $\mathrm{Na}^{+}$complexes, J. Phys. Chem. A 105, 769 (2001). 